\title{
Commutators of Singular Integral Operators Related to Magnetic Schrödinger Operators
}

\author{
Wanqing Ma and Yu Liu* \\ School of Mathematics and Physics, University of Science and Technology Beijing, \\ Beijing 100083, China
}

Received 7 December 2016; Accepted (in revised version) 7 September 2017

\begin{abstract}
Let $A:=-(\nabla-i \vec{a}) \cdot(\nabla-i \vec{a})+V$ be a magnetic Schrödinger operator on $L^{2}\left(\mathbb{R}^{n}\right)$, $n \geq 2$, where $\vec{a}:=\left(a_{1}, \cdots, a_{n}\right) \in L_{\text {loc }}^{2}\left(\mathbb{R}^{n}, \mathbb{R}^{n}\right)$ and $0 \leq V \in L_{\text {loc }}^{1}\left(\mathbb{R}^{n}\right)$. In this paper, we show that for a function $b$ in Lipschitz space $\operatorname{Lip}_{\alpha}\left(\mathbb{R}^{n}\right)$ with $\alpha \in(0,1)$, the commutator $\left[b, V^{1 / 2} A^{-1 / 2}\right]$ is bounded from $L^{p}\left(\mathbb{R}^{n}\right)$ to $L^{q}\left(\mathbb{R}^{n}\right)$, where $p, q \in(1,2]$ and $1 / p-1 / q=$ $\alpha / n$.
\end{abstract}

Key Words: Commutator, Lipschitz space, the sharp maxical function, magnetic Schrödinger operator, Hölder inequality.

AMS Subject Classifications: 42B20, 42B35

\section{Introduction}

Let $b$ be a locally integrable function on $\mathbb{R}^{n}$ and $T$ be a linear operator. For a suitable function $f$, the commutator is defined by $[b, T] f=b T(f)-T(b f)$. It is well known that Coifman, Rochberg and Weiss [3] proved that $[b, T]$ is a bounded operator on $L^{p}$ for $1<$ $p<\infty$ if and only if $b \in \mathrm{BMO}\left(\mathbb{R}^{n}\right)$, when $T$ is a Calderón-Zygmund operator. Janson [4] proved that the commutator $[b, T]$ is bounded from $L^{p}\left(\mathbb{R}^{n}\right)$ into $L^{q}\left(\mathbb{R}^{n}\right), 1<p<q<\infty$, if and only if $b \in \operatorname{Lip}_{\alpha}\left(\mathbb{R}^{n}\right)$ with $\alpha=\left(\frac{1}{p}-\frac{1}{q}\right) n$, where the Lipschitz space $\operatorname{Lip}_{\alpha}\left(\mathbb{R}^{n}\right)$ consists of the functions $f$ satisfying

$$
\|f\|_{\operatorname{Lip}_{\alpha}}:=\sup _{x, y \in \mathbb{R}^{n}, x \neq y} \frac{|f(x)-f(y)|}{|x-y|^{\alpha}}<\infty, \quad 0<\alpha<1 .
$$

Furthermore, $\mathrm{Lu}, \mathrm{Wu}$ and Yang studied the boundedness properties of the commutator $[b, T]$ on the classical Hardy spaces when $b \in \operatorname{Lip}_{\alpha}\left(\mathbb{R}^{n}\right)$ in [12].

In recent years, more scholars pay attention to the boundedness of the commutators $[b, T]$ when $T$ are the singular integral operators associated with the Schrödinger operator

${ }^{*}$ Corresponding author. Email addresses: 2424077889@qq. com (W. Q. Ma), 1iuyu75@pku. org. cn (Y. Liu) 
(cf. [1,6-11]). When the potential $V$ satisfies the weaker condition, the operator $T$ may not be a Calderón-Zygmund operator. In this paper we focus on the boundedness of the commutators $[b, T]$ when $T$ are the singular integral operators associated with the magnetic Schrödinger operator based on the research in [5] and [16].

Consider a real vector potential $\vec{a}=\left(a_{1}, \cdots, a_{n}\right)$ and an electric potential $V$. In this paper, we assume that

$$
\begin{aligned}
& a_{k} \in L_{\mathrm{loc}}^{2}\left(\mathbb{R}^{n}\right), \quad \forall k=1, \cdots, n, \\
& 0 \leq V \in L_{\mathrm{loc}}^{1}\left(\mathbb{R}^{n}\right) .
\end{aligned}
$$

Let $L_{k}=\partial / \partial x_{k}-i a_{k}$. We adopt the same notation as in [5] and define the sesquilinear form $Q$ by

$$
Q(f, g):=\sum_{k=1}^{n} \int_{\mathbb{R}^{n}} L_{k} f \overline{L_{k} g} d x+\int_{\mathbb{R}^{n}} V f \bar{g} d x,
$$

with domain

$$
D(Q):=\left\{f \in L^{2}\left(\mathbb{R}^{n}\right): L_{k} f \in L^{2}\left(\mathbb{R}^{n}\right), k \in 1, \cdots, n, \sqrt{V} f \in L^{2}\left(\mathbb{R}^{n}\right)\right\} .
$$

It is known that $Q$ is closed and symmetric. So the magnetic Schrödinger operator $A$ is a self-adjoint operator associated with $Q$.

The domain of $A$ is given by

$$
D(A)=\left\{f \in D(Q), \exists g \in L^{2}\left(\mathbb{R}^{n}\right) \text { such that } Q(f, \varphi)=\int_{\mathbb{R}^{n}} g \bar{\varphi} d x, \forall \varphi \in D(Q)\right\},
$$

and $A$ is formally given by the following expression

$$
A f=\sum_{k=1}^{n} L_{k}^{*} L_{k} f+V f
$$

or $A=-(\nabla-i \vec{a}) \cdot(\nabla-i \vec{a})+V$, where $L_{k}^{*}$ is the adjoint operator of $L_{k}$. For $k=1, \cdots, n$, the operators $L_{k} A^{-1 / 2}$ and $V^{1 / 2} A^{-1 / 2}$ are called the Riesz transforms associated with $A$. Moreover, it was proved in [14] that for each $k=1, \cdots, n$, the Riesz transform $L_{k} A^{-1 / 2}$ and $V^{1 / 2} A^{-1 / 2}$ are bounded on $L^{p}\left(\mathbb{R}^{n}\right)$ for all $1<p \leq 2$. Namely, there exists a constant $C>0$ such that

$$
\left\|V^{1 / 2} A^{-1 / 2} f\right\|_{L^{p}\left(\mathbb{R}^{n}\right)}+\sum_{k=1}^{n}\left\|L_{k} A^{-1 / 2} f\right\|_{L^{p}\left(\mathbb{R}^{n}\right)} \leq C\|f\|_{L^{p}\left(\mathbb{R}^{n}\right)}, \quad 1<p \leq 2 .
$$

Furthermore, in [5] Duong and Yan proved that the commutators $\left[b, V^{1 / 2} A^{-1 / 2}\right]$ and $\left[b, L_{k} A^{-1 / 2}\right]$ are bounded on $L^{p}$ for $1<p \leq 2$, that is, there exists a constant $C>0$ such that

$$
\left\|\left[b, V^{1 / 2} A^{-1 / 2}\right] f\right\|_{L^{p}\left(\mathbb{R}^{n}\right)}+\left\|\left[b, L_{k} A^{-1 / 2}\right] f\right\|_{L^{p}\left(\mathbb{R}^{n}\right)} \leq C\|f\|_{L^{p}\left(\mathbb{R}^{n}\right)}, \quad \text { where } b \in \operatorname{BMO}\left(\mathbb{R}^{n}\right) .
$$


See also Shen's result in [15] for $L^{p}$-boundedness of singular integral operators related to the magnetic Schrödinger operator, which is different from the operators $L_{k} A^{-1 / 2}$ and $V^{1 / 2} A^{-1 / 2}$. Recently, D. Y. Yang in [16] has proven that for $k \in\{1, \cdots, n\}$, the commutators $\left[b, L_{k} A^{-1 / 2}\right]$ are bounded from $L^{p}\left(\mathbb{R}^{n}\right)$ to $L^{q}\left(\mathbb{R}^{n}\right)$ with $1 / p-1 / q=\alpha / n$, where $b \in$ $\operatorname{Lip}_{\alpha}\left(\mathbb{R}^{n}\right)$. Inspired by [5] and [16], the purpose of this paper is to study the boundedness

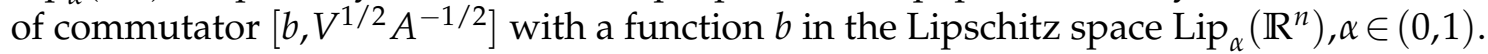

We are now in a position to give our main result, which will be proven in the next section.

Theorem 1.1. Let $\alpha \in(0,1), p, q \in(1,2]$ with $1 / p-1 / q=\alpha / n$. Assume that $b \in \operatorname{Lip}_{\alpha}\left(\mathbb{R}^{n}\right)$. Then the commutator $\left[b, V^{1 / 2} A^{-1 / 2}\right]$ is bounded from $L^{p}\left(\mathbb{R}^{n}\right)$ to $L^{q}\left(\mathbb{R}^{n}\right)$.

\section{Proof of Theorem 1.1}

In this section, we adopt the method in [16] to prove Theorem 1.1. Firstly, we begin with the sharp maximal function $M_{A}^{\#}$ established in [13]. For any $f \in L^{p}\left(\mathbb{R}^{n}\right), p \in[1, \infty)$, the sharp maximal function $M_{A}^{\#}$ associated with the semigroup $\left\{e^{-t A}\right\}$ is given by

$$
M_{A}^{\#}(f)(x):=\sup _{x \in B} \frac{1}{|B|} \int_{B}\left|f(y)-e^{-t_{B} A} f(y)\right| d y,
$$

where $r_{B}$ is the radius of the ball $B$ and $t_{B}:=r_{B}^{2}$.

Lemma 2.1. Let $p \in(1, \infty)$. There exists a positive constant $C_{p}$ such that for all $f \in L^{p}\left(\mathbb{R}^{n}\right)$,

$$
\|f\|_{L^{p}\left(\mathbb{R}^{n}\right)} \leq C_{p}\left\|M_{A}^{\#}(f)\right\|_{L^{p}\left(\mathbb{R}^{n}\right)} .
$$

Proof of Theorem 1.1. Now, we prove the boundedness of the commutator $\left[b, V^{1 / 2} A^{-1 / 2}\right]$ in Theorem 1.1. Let $\left(V^{1 / 2} A^{-1 / 2}\right)^{*}=A^{-1 / 2} V^{1 / 2}$ denote the adjoint operator of $V^{1 / 2} A^{-1 / 2}$. By duality, for given $p, q \in(1,2]$ with $1 / p-1 / q=\alpha / n$, it suffices to prove $\left[b, A^{-1 / 2} V^{1 / 2}\right]$ is bounded from $L^{q^{\prime}}\left(\mathbb{R}^{n}\right)$ to $L^{p^{\prime}}\left(\mathbb{R}^{n}\right)$. To obtain the conclusion, it suffices to prove that there exists a constant $C$ such that for all $f \in C_{c}^{\infty}\left(\mathbb{R}^{n}\right)$ and $x \in \mathbb{R}^{n}$,

$$
M_{A}^{\#}\left(\left[b, A^{-1 / 2} V^{1 / 2}\right] f\right)(x) \leq C\|b\|_{\operatorname{Lip}_{\alpha}\left(\mathbb{R}^{n}\right)}\left[M_{2, \alpha}\left(A^{-1 / 2} V^{1 / 2} f\right)(x)+M_{2, \alpha}(f)(x)\right],
$$

where for $r \in[2, n / \alpha)$ and any suitable function $f$,

$$
M_{r, \alpha}(f)(x):=\sup _{x \in B} \frac{1}{|B|^{-\alpha / n}}\left(\frac{1}{|B|} \int_{B}|f(y)|^{r} d y\right)^{1 / r} .
$$

In fact, assume that (2.1) holds. For $b \in \operatorname{Lip}_{\alpha}\left(\mathbb{R}^{n}\right)$ and each $N \in \mathbb{N}$, define $b_{N}:=$ $\min \{N,|b|\} \operatorname{sgn}(b)$. Then we conclude that $b_{N} \in L^{\infty}\left(\mathbb{R}^{n}\right)$ and $\left\|b_{N}\right\|_{\operatorname{Lip}_{\alpha}\left(\mathbb{R}^{n}\right)} \leq C\|b\|_{\operatorname{Lip}_{\alpha}\left(\mathbb{R}^{n}\right)}$, where $C$ is a constant. Moreover, it has been proved in Theorem 1.1 of [14] that $V^{1 / 2} A^{-1 / 2}$ 
is bounded on $L^{p}\left(\mathbb{R}^{n}\right)$ for all $1<p \leq 2$. So $A^{-1 / 2} V^{1 / 2}$ is bounded on $L^{s}\left(\mathbb{R}^{n}\right)$ for $s \in[2, \infty)$, then we see that for all $f \in C_{c}^{\infty}\left(\mathbb{R}^{n}\right),\left[b_{N}, A^{-1 / 2} V^{1 / 2}\right](f) \in L^{S}\left(\mathbb{R}^{n}\right)$ and

$$
\left\|\left[b_{N}, A^{-1 / 2} V^{1 / 2}\right](f)\right\|_{L^{s}\left(\mathbb{R}^{n}\right)} \leq N\|f\|_{L^{s}\left(\mathbb{R}^{n}\right)} .
$$

Recall that $M_{2, \alpha}$ is bounded from $L^{s}\left(\mathbb{R}^{n}\right)$ to $L^{t}\left(\mathbb{R}^{n}\right)$ with $s \in(2, n / \alpha)$ and $1 / s-1 / t=$ $\alpha / n$, see Chanillo [2]. By this fact together with $1 / q^{\prime}-1 / p^{\prime}=\alpha / n$, Lemma 2.1 and (2.1), we have that for all $f \in C_{c}^{\infty}\left(\mathbb{R}^{n}\right)$,

$$
\begin{aligned}
& \left\|\left[b_{N}, A^{-1 / 2} V^{1 / 2}\right](f)\right\|_{L^{p^{\prime}}\left(\mathbb{R}^{n}\right)} \\
\leq & C\left\|M_{A}^{\#}\left(\left[b_{N}, A^{-1 / 2} V^{1 / 2}\right](f)\right)\right\|_{L^{p^{\prime}}\left(\mathbb{R}^{n}\right)} \\
\leq & \left.C\left\|b_{N}\right\|_{\operatorname{Lip}_{\alpha}\left(\mathbb{R}^{n}\right)} \| M_{2, \alpha}\left(A^{-1 / 2} V^{1 / 2} f\right)(x)\right)+M_{2, \alpha}(f)(x) \|_{L^{p^{\prime}}\left(\mathbb{R}^{n}\right)} \\
\leq & C\|b\|_{\operatorname{Lip}_{\alpha}\left(\mathbb{R}^{n}\right)}\|f\|_{L^{q^{\prime}}\left(\mathbb{R}^{n}\right)} .
\end{aligned}
$$

A standard argument together with the Fatou lemma then implies that for all $f \in L^{q^{\prime}}\left(\mathbb{R}^{n}\right)$,

$$
\left[b, A^{-1 / 2} V^{1 / 2}\right](f) \in L^{p^{\prime}}\left(\mathbb{R}^{n}\right)
$$

and

$$
\left\|\left[b, A^{-1 / 2} V^{1 / 2}\right](f)\right\|_{L^{p^{\prime}}\left(\mathbb{R}^{n}\right)} \leq C\|b\|_{\operatorname{Lip}_{\alpha}\left(\mathbb{R}^{n}\right)}\|f\|_{L^{q^{\prime}}\left(\mathbb{R}^{n}\right)^{\prime}}
$$

which imply Theorem 1.1.

Now, we prove (2.1) is valid. For any $f \in L^{q^{\prime}}\left(\mathbb{R}^{n}\right)$, and $x \in \mathbb{R}^{n}$, choose a ball $B:=$ $B\left(x_{B}, r_{B}\right)=\left\{y \in \mathbb{R}^{n}:\left|x_{B}-y\right|<r_{B}\right\}$ which contains $x$. Set $T:=A^{-1 / 2} V^{1 / 2}$. Let $f_{1}=f \chi_{2 B}$ and $f_{2}:=f-f_{1}$. Then we have the following decompositions:

$$
[b, T] f=\left(b-b_{B}\right) T f-T\left(\left(b-b_{B}\right) f_{1}\right)-T\left(\left(b-b_{B}\right) f_{2}\right)
$$

and

$$
e^{-t_{B} A}([b, T] f)=e^{-t_{B} A}\left(\left(b-b_{B}\right) T f\right)-e^{-t_{B} A} T\left(\left(b-b_{B}\right) f_{1}\right)-e^{-t_{B} A} T\left(\left(b-b_{B}\right) f_{2}\right)
$$

for any function $f$ and ball $B$, where

$$
f_{B}:=\frac{1}{|B|} \int_{B} f(z) d z, \quad t_{B}=r_{B}^{2}
$$


Therefore,

$$
\begin{aligned}
& \frac{1}{|B|} \int_{B}\left|\left(I-e^{-t_{B} A}\right)[b, T] f(y)\right| d y \\
\leq & \frac{1}{|B|} \int_{B}\left|\left(b-b_{B}\right) T f(y)\right| d y+\frac{1}{|B|} \int_{B}\left|T\left(\left(b-b_{B}\right) f_{1}\right)(y)\right| d y \\
& +\frac{1}{|B|} \int_{B}\left|e^{-t_{B} A}\left(\left(b-b_{B}\right) T f\right)(y)\right| d y+\frac{1}{|B|} \int_{B}\left|e^{-t_{B} A} T\left(\left(b-b_{B}\right) f_{1}\right)(y)\right| d y \\
& +\frac{1}{|B|} \int_{B}\left|\left(I-e^{-t_{B} A}\right) T\left(\left(b-b_{B}\right) f_{2}\right)(y)\right| d y \\
:= & \mathrm{I}+\mathrm{II}+\mathrm{III}+\mathrm{IV}+\mathrm{V} .
\end{aligned}
$$

Firstly, we get

$$
\begin{aligned}
& \left|b(y)-b_{B}\right|=\left|b(y)-\frac{1}{|B|} \int_{B} b(z) d z\right|=\left|\frac{1}{|B|} \int_{B}(b(y)-b(z)) d z\right| \\
& \leq \frac{1}{|B|} \int_{B}|y-z|^{\alpha} \frac{|b(y)-b(z)|}{|y-z|^{\alpha}} d z \\
& \leq\|b\|_{\operatorname{Lip}_{\alpha}\left(\mathbb{R}^{n}\right)}\left|\frac{1}{|B|} \int_{B}\right| y-\left.z\right|^{\alpha} d z \\
& \leq C|B|^{\alpha / n}\|b\|_{\operatorname{Lip}_{\alpha}\left(\mathbb{R}^{n}\right)} .
\end{aligned}
$$

For I, by the Hölder inequality and (2.3), we have

$$
\begin{aligned}
\mathrm{I} & =\frac{1}{|B|} \int_{B}\left|\left(b-b_{B}\right) T f(y)\right| d y \\
& \leq\left(\frac{1}{|B|} \int_{B}\left|b(y)-b_{B}\right|^{2} d y\right)^{1 / 2}\left(\frac{1}{|B|} \int_{B}|T f(y)|^{2} d y\right)^{1 / 2} \\
& \leq C\|b\|_{\operatorname{Lip}_{\alpha}\left(\mathbb{R}^{n}\right)} M_{2, \alpha}(T f)(x) \\
& =C\|b\|_{\operatorname{Lip}_{\alpha}\left(\mathbb{R}^{n}\right) M_{2, \alpha}\left(A^{-1 / 2} V^{1 / 2} f\right)(x) .}
\end{aligned}
$$

For II, using the Hölder inequality again and the $L^{2}\left(\mathbb{R}^{n}\right)$-boundedness of $T$, if follows that

$$
\begin{aligned}
\mathrm{II} & =\frac{1}{|B|} \int_{B}\left|T\left(\left(b-b_{B}\right) f_{1}\right)(y)\right| d y \\
& \leq\left(\frac{1}{|B|} \int_{B}\left|T\left(\left(b-b_{B}\right) f_{1}\right)(y)\right|^{2} d y\right)^{1 / 2} \\
& \leq C\left(\frac{1}{|B|} \int_{2 B}\left|b(y)-b_{B}\right|^{2}|f(y)|^{2} d y\right)^{1 / 2} \\
& \leq C\|b\|_{\operatorname{Lip}_{\alpha}\left(\mathbb{R}^{n}\right)} \frac{1}{|B|^{-\alpha / n}}\left(\frac{1}{|B|} \int_{2 B}|f(y)|^{2} d y\right)^{1 / 2} \\
& \leq C\|b\|_{\operatorname{Lip}_{\alpha}\left(\mathbb{R}^{n}\right)} M_{2, \alpha}(f)(x) .
\end{aligned}
$$


To estimate III, it follows from [5] that the kernel $p_{t}(y, z)$ of $e^{-t A}$ satisfies that for all $t>0$ and almost all $y, z \in \mathbb{R}^{n}$,

$$
p_{t}(y, z) \leq(4 \pi t)^{-\frac{n}{2}} \exp \left(-\frac{|y-z|^{2}}{4 t}\right) .
$$

Let $g:=\left(b-b_{B}\right) T f$. By (2.4), the formula equation of $e^{-|x|} \leq C|x|^{-N}$ and the conclusion of $\mathrm{I}$, for any $y \in B$,

$$
\begin{aligned}
& \left|e^{-t_{B} A} g(y)\right| \leq \int_{\mathbb{R}^{n}}\left|p_{t_{B}}(y, z) g(z)\right| d z \leq \int_{\mathbb{R}^{n}}\left|p_{t_{B}}(y, z)\right||g(z)| d z \\
& \leq \int_{\mathbb{R}^{n}} t_{B}^{-\frac{n}{2}} \exp \left(-\frac{|y-z|^{2}}{4 t_{B}}\right)|g(z)| d z \\
& =\int_{|y-z|<2 t_{B}^{1 / 2}} t_{B}^{-\frac{n}{2}} \exp \left(-\frac{|y-z|^{2}}{4 t_{B}}\right)|g(z)| d z \\
& +\sum_{k=0}^{\infty} \int_{2^{k} t_{B}^{1 / 2} \leq|y-z|<2^{k+1} t_{B}^{1 / 2}} t_{B}^{-\frac{n}{2}} \exp \left(-\frac{|y-z|^{2}}{4 t_{B}}\right)|g(z)| d z \\
& \leq C\left[\frac{1}{|B|} \int_{B}|g(z)| d z+\sum_{k=0}^{\infty} \frac{1}{t_{B}^{\frac{n}{2}}} \int_{2^{k} t_{B}^{1 / 2} \leq|y-z|<2^{k+1} t_{B}^{1 / 2}}\left(\frac{t_{B}}{|y-z|^{2}}\right)^{N}|g(z)| d z\right] \\
& \leq C\left[\frac{1}{|B|} \int_{B}|g(z)| d z+\sum_{k=0}^{\infty} \frac{1}{t_{B}^{\frac{n}{2}}} \frac{t_{B}^{N}}{\left(2^{k} t_{B}^{1 / 2}\right)^{2 N}} \int_{|y-z|<2^{k+1} t_{B}^{1 / 2}}|g(z)| d z\right] \\
& \leq C\left[\|b\|_{\operatorname{Lip}_{\alpha}\left(\mathbb{R}^{n}\right)} M_{2, \alpha}\left(A^{-1 / 2} V^{1 / 2} f\right)(x)\right. \\
& \left.+\sum_{k=0}^{\infty} \frac{1}{2^{k(2 N-n)}}\|b\|_{\operatorname{Lip}_{\alpha}\left(\mathbb{R}^{n}\right)} M_{2, \alpha}\left(A^{-1 / 2} V^{1 / 2} f\right)(x)\right] \\
& \leq C\|b\|_{\operatorname{Lip}_{\alpha}\left(\mathbb{R}^{n}\right)} M_{2, \alpha}\left(A^{-1 / 2} V^{1 / 2} f\right)(x),
\end{aligned}
$$

where $N>n / 2$. So for III, it is easy for us to get

$$
\begin{aligned}
\mathrm{III} & =\frac{1}{|B|} \int_{B}\left|e^{-t_{B} A}\left(\left(b-b_{B}\right) T f\right)(y)\right| d y \\
& =\frac{1}{|B|} \int_{B}\left|e^{-t_{B} A} g(y)\right| d y \\
& \leq C\|b\|_{\operatorname{Lip}_{\alpha}\left(\mathbb{R}^{n}\right)} M_{2, \alpha}\left(A^{-1 / 2} V^{1 / 2} f\right)(x) .
\end{aligned}
$$

For IV, for all locally integrable functions $f$ and $x \in \mathbb{R}^{n}$, let $M(f)(x)$ be the Hardy-Littlewood maximal function as follow:

$$
M(f)(x):=\sup _{x \in B} \frac{1}{|B|} \int_{B}|f(y)| d y .
$$


By (2.4), the conclusion of II, the Hölder inequality and the $L^{2}\left(\mathbb{R}^{n}\right)$-boundedness of $T$ and $M$, we conclude that

$$
\begin{aligned}
\mathrm{IV} & =\frac{1}{|B|} \int_{B}\left|e^{-t_{B} A} T\left(\left(b-b_{B}\right) f_{1}\right)(y)\right| d y \\
& \leq C \frac{1}{|B|} \int_{B} M\left[T\left(\left(b-b_{B}\right) f_{1}\right)\right](y) d y \\
& \leq C\left[\frac{1}{|B|} \int_{B}\left\{M\left[T\left(\left(b-b_{B}\right) f_{1}\right)\right](y)\right\}^{2} d y\right]^{1 / 2} \\
& \leq C\left[\frac{1}{|B|} \int_{B}\left|T\left(\left(b-b_{B}\right) f_{1}\right)(y)\right|^{2} d y\right]^{1 / 2} \\
& \leq C\left[\frac{1}{|B|} \int_{2 B}\left|\left(b(y)-b_{B}\right) f(y)\right|^{2} d y\right]^{1 / 2} \\
& \leq C\|b\|_{\operatorname{Lip}_{\alpha}\left(\mathbb{R}^{n}\right) M_{2, \alpha}(f)(x) .}
\end{aligned}
$$

In order to estimate the term $\mathrm{V}$, we need the following Proposition 2.1 and Lemma 2.2.

Proposition 2.1 (cf. Proposition 3.1 in [5]). Fix $s>0$. Let $A=-(\nabla-i \vec{a}) \cdot(\nabla-i \vec{a})+V$ be the magnetic Schrödinger operator. Then for any $m \in \mathbb{N}$, there exist positive constants $C$ and $c$ such that

$$
\int_{2^{m} \sqrt{t} \leq|x-y|<2^{m+1} \sqrt{t}}\left(\left|V^{1 / 2} p_{s}(x, y)\right|^{2}+\sum_{k=1}^{n}\left|L_{k} p_{s}(x, y)\right|^{2}\right) d x \leq C s^{-n}\left(2^{m} \sqrt{t}\right)^{n-2} \exp \left(-\frac{2^{2 m} t}{c s}\right)
$$

for all $s>0$ and $y \in \mathbb{R}^{n}$.

Lemma 2.2. For a real vector potential $\vec{a}=\left(a_{1}, \cdots, a_{n}\right)$ and an electric potential $V$, we assume that $a_{k} \in L_{\text {loc }}^{2}\left(\mathbb{R}^{n}, \mathbb{R}^{n}\right), \forall k=1, \cdots, n, 0 \leq V \in L_{\text {loc }}^{1}\left(\mathbb{R}^{n}\right)$. Then the composite operator (I$\left.e^{-t A}\right) A^{-1 / 2} V^{1 / 2}, t>0$, which is the adjoint operator of $V^{1 / 2} A^{-1 / 2}\left(I-e^{-t A}\right)$, has an associated kernel $K_{t}^{*}(y, z)$ which satisfies

$$
\sum_{m=0}^{\infty} 2^{m}\left(2^{m} \sqrt{t}\right)^{n / 2}\left(\int_{2^{m} \sqrt{t} \leq|y-z|<2^{m+1} \sqrt{t}}\left|K_{t}^{*}(y, z)\right|^{2} d y\right)^{1 / 2} \leq C<\infty, \quad z \in \mathbb{R}^{n}
$$

Proof of Lemma 2.2. Firstly, we need to compute the $K_{t}^{*}(y, z)$ of $\left(I-e^{-t A}\right) A^{-1 / 2} V^{1 / 2}$. Observe that

$$
A^{-1 / 2}=\frac{1}{\sqrt{\pi}} \int_{0}^{\infty} e^{-s A} \frac{d s}{\sqrt{s}},
$$


then we have

$$
\begin{aligned}
& \left(I-e^{-t A}\right) A^{-1 / 2} V^{1 / 2} \\
= & \frac{1}{\sqrt{\pi}} \int_{0}^{\infty} e^{-s A} V^{1 / 2} \frac{d s}{\sqrt{s}}-\frac{1}{\sqrt{\pi}} \int_{0}^{\infty} e^{-(s+t) A} V^{1 / 2} \frac{d s}{\sqrt{s}} \\
= & \frac{1}{\sqrt{\pi}} \int_{0}^{\infty} e^{-s A} V^{1 / 2} \frac{d s}{\sqrt{s}}-\frac{1}{\sqrt{\pi}} \int_{t}^{\infty} e^{-s A} V^{1 / 2} \frac{d s}{\sqrt{s-t}} \\
= & \frac{1}{\sqrt{\pi}} \int_{0}^{\infty} e^{-s A} V^{1 / 2} \frac{d s}{\sqrt{s}}-\frac{1}{\sqrt{\pi}} \int_{0}^{\infty} e^{-s A} V^{1 / 2} \frac{\chi(s>t)}{\sqrt{s-t}} d s \\
= & \frac{1}{\sqrt{\pi}} \int_{0}^{\infty}\left(\frac{1}{\sqrt{s}}-\frac{\chi(s>t)}{\sqrt{s-t}}\right) e^{-s A} V^{1 / 2} d s .
\end{aligned}
$$

Therefore,

$$
\left|K_{t}^{*}(y, z)\right|=\left|\frac{1}{\sqrt{\pi}} \int_{0}^{\infty}\left(\frac{1}{\sqrt{s}}-\frac{\chi_{(s>t)}}{\sqrt{s-t}}\right) p_{s}(y, z) V^{1 / 2} d s\right| .
$$

By Minkowski's inequality, we have

$$
\begin{aligned}
& \left(\int_{2^{m} \sqrt{t} \leq|y-z|<2^{m+1} \sqrt{t}}\left|K_{t}^{*}(y, z)\right|^{2} d y\right)^{1 / 2} \\
\leq & \frac{1}{\sqrt{\pi}} \int_{0}^{\infty}\left|\frac{1}{\sqrt{s}}-\frac{\chi(s>t)}{\sqrt{s-t}}\right|\left(\int_{2^{m} \sqrt{t} \leq|y-z|<2^{m+1} \sqrt{t}}\left|p_{s}(y, z) V^{1 / 2}\right|^{2} d y\right)^{1 / 2} d s .
\end{aligned}
$$

Together with Proposition 2.1, this gives

$$
\begin{aligned}
& \sum_{m=0}^{\infty} 2^{m}\left(2^{m} \sqrt{t}\right)^{n / 2}\left(\int_{2^{m} \sqrt{t} \leq|y-z|<2^{m+1} \sqrt{t}}\left|K_{t}^{*}(y, z)\right|^{2} d y\right)^{1 / 2} \\
\leq & C \sum_{m=0}^{\infty} 2^{m}\left(2^{m} \sqrt{t}\right)^{n / 2} \int_{0}^{\infty}\left|\frac{1}{\sqrt{s}}-\frac{\chi_{(s>t)}}{\sqrt{s-t}}\right|\left(\int_{2^{m} \sqrt{t} \leq|y-z|<2^{m+1} \sqrt{t}}\left|p_{s}(y, z) V^{1 / 2}\right|^{2} d y\right)^{1 / 2} d s \\
\leq & C \sum_{m=0}^{\infty} 2^{m}\left(2^{m} \sqrt{t}\right)^{n / 2} \int_{0}^{\infty}\left|\frac{1}{\sqrt{s}}-\frac{\chi_{(s>t)}}{\sqrt{s-t}}\right| s^{-n / 2}\left(2^{m} \sqrt{t}\right)^{(n-2) / 2} \exp \left(-\frac{2^{2 m} t}{2 c s}\right) d s \\
= & C \sum_{m=0}^{\infty} 2^{m} \int_{0}^{t}\left|\frac{1}{\sqrt{s}}-\frac{\chi_{(s>t)}}{\sqrt{s-t}}\right| s^{-n / 2}\left(2^{m} \sqrt{t}\right)^{n-1} \exp \left(-\frac{2^{2 m} t}{2 c s}\right) d s \\
& +C \sum_{m=0}^{\infty} 2^{m} \int_{t}^{\infty}\left|\frac{1}{\sqrt{s}}-\frac{\chi_{(s>t)}}{\sqrt{s-t}}\right| s^{-n / 2}\left(2^{m} \sqrt{t}\right)^{n-1} \exp \left(-\frac{2^{2 m} t}{2 c s}\right) d s \\
:= & \mathrm{I}_{1}+\mathrm{II}_{1} .
\end{aligned}
$$

We first estimate the term $\mathrm{I}_{1}$. Note that $\chi_{s>t} \equiv 0$ for $s<t$. This, together with the fact 
that $\omega^{\beta} e^{-c \omega} \leq C$ for any $\omega, \beta>0$, shows

$$
\begin{aligned}
\mathrm{I}_{1} & =C \sum_{m=0}^{\infty} 2^{m} \int_{0}^{t}\left|\frac{1}{\sqrt{s}}-\frac{\chi(s>t)}{\sqrt{s-t}}\right| s^{-n / 2}\left(2^{m} \sqrt{t}\right)^{n-1} \exp \left(-\frac{2^{2 m} t}{2 c s}\right) d s \\
& =C \sum_{m=0}^{\infty} 2^{m} \int_{0}^{t} \mid s^{-(n+1) / 2}\left(2^{m} \sqrt{t}\right)^{n-1} \exp \left(-\frac{2^{2 m} t}{2 c s}\right) d s \\
& \leq C \sum_{m=0}^{\infty} 2^{m} \int_{0}^{t}\left(\frac{2^{2 m} t}{s}\right)^{\frac{n-1}{2}}\left(\frac{s}{2^{2 m} t}\right)^{\frac{n-1}{2}+1} \frac{d s}{s} \\
& \leq C \sum_{m=0}^{\infty} 2^{-m} t^{-1} \int_{0}^{t} d s \leq C^{\prime}<\infty .
\end{aligned}
$$

Consider the term $\mathrm{II}_{1}$. Oberve that, for $s>t$, then $\chi_{s>t}=1$. A direct calculation shows that

$$
\begin{aligned}
\left|\frac{1}{\sqrt{s}}-\frac{\chi(s>t)}{\sqrt{s-t}}\right| & =\left|\frac{1}{\sqrt{s}}-\frac{1}{\sqrt{s-t}}\right|=\frac{1}{\sqrt{s} \sqrt{s-t}} \times|\sqrt{s-t}-\sqrt{s}| \\
& =\frac{1}{\sqrt{s} \sqrt{s-t}} \times \frac{t}{\sqrt{s-t}+\sqrt{s}}=\frac{t}{s \sqrt{s-t}+\sqrt{s}(s-t)} \\
& \leq \frac{t}{s \sqrt{s-t}} .
\end{aligned}
$$

Substituting the above into the term $\mathrm{II}_{1}$, we obtain

$$
\begin{aligned}
\mathrm{II}_{1}= & C \sum_{m=0}^{\infty} 2^{m} \int_{t}^{\infty}\left|\frac{1}{\sqrt{s}}-\frac{\chi(s>t)}{\sqrt{s-t}}\right| s^{-n / 2}\left(2^{m} \sqrt{t}\right)^{n-1} \exp \left(-\frac{2^{2 m} t}{2 c s}\right) d s \\
\leq & C \sum_{m=0}^{\infty} 2^{m} \int_{t}^{\infty} \frac{t}{s \sqrt{s-t}} s^{-n / 2}\left(2^{m} \sqrt{t}\right)^{n-1} \exp \left(-\frac{2^{2 m} t}{2 c s}\right) d s \\
= & C \sum_{m=0}^{\infty} 2^{m} \int_{t}^{2 t} \frac{t}{\sqrt{s} \sqrt{s-t}}\left(\frac{2^{2 m} t}{s}\right)^{\frac{n-1}{2}} \exp \left(-\frac{2^{2 m} t}{2 c s}\right) \frac{d s}{s} \\
& +C \sum_{m=0}^{\infty} 2^{m} \int_{2 t}^{\infty} \frac{t}{\sqrt{s} \sqrt{s-t}}\left(\frac{2^{2 m} t}{s}\right)^{\frac{n-1}{2}} \exp \left(-\frac{2^{2 m} t}{2 c s}\right) \frac{d s}{s} \\
:= & \mathrm{II}_{1,1}+\mathrm{II}_{1,2} .
\end{aligned}
$$

Because that $t<s \leq 2 t$, so we can get

$$
\left(\frac{2^{2 m} t}{s}\right)^{\frac{n-1}{2}} \leq 2^{m(n-1)}, \quad \exp \left(-\frac{2^{2 m} t}{2 c s}\right) \leq \exp \left(-\frac{2^{2 m}}{4 c}\right), \quad \frac{t}{s \sqrt{s}}<t^{-1 / 2}
$$


Therefore,

$$
\begin{aligned}
\mathrm{II}_{1,1} & =C \sum_{m=0}^{\infty} 2^{m} \int_{t}^{2 t} \frac{t}{\sqrt{s} \sqrt{s-t}}\left(\frac{2^{2 m} t}{s}\right)^{\frac{n-1}{2}} \exp \left(-\frac{2^{2 m} t}{2 c s}\right) \frac{d s}{s} \\
& \leq C \sum_{m=0}^{\infty} 2^{m} \int_{t}^{2 t} \frac{t^{-1 / 2}}{\sqrt{s-t}} 2^{m(n-1)} \exp \left(-\frac{2^{2 m}}{4 c}\right) d s \\
& \leq C \sum_{m=0}^{\infty} 2^{m n} \exp \left(-\frac{2^{2 m}}{4 c}\right) t^{-1 / 2} \int_{t}^{2 t} \frac{1}{\sqrt{s-t}} d s \\
& \leq C \sum_{m=0}^{\infty} 2^{m n} \exp \left(-\frac{2^{2 m}}{4 c}\right) \leq C^{\prime}<\infty .
\end{aligned}
$$

Finally, we estimate the term $\mathrm{II}_{1,2}$. Since $s>2 t$, we have that $\sqrt{s-t}>\sqrt{s} / 2$. Hence,

$$
\begin{aligned}
\mathrm{II}_{1,2} & =C \sum_{m=0}^{\infty} 2^{m} \int_{2 t}^{\infty} \frac{t}{\sqrt{s} \sqrt{s-t}}\left(\frac{2^{2 m} t}{s}\right)^{\frac{n-1}{2}} \exp \left(-\frac{2^{2 m} t}{2 c s}\right) \frac{d s}{s} \\
& \leq C \sum_{m=0}^{\infty} 2^{m} \int_{2 t}^{\infty} \frac{t}{s}\left(\frac{2^{2 m} t}{s}\right)^{\frac{n-1}{2}} \exp \left(-\frac{2^{2 m} t}{2 c s}\right) \frac{d s}{s} \\
& \leq C \sum_{m=0}^{\infty} 2^{m} \int_{2 t}^{\infty} \frac{t}{s}\left(\frac{2^{2 m} t}{s}\right)^{\frac{n-1}{2}}\left(\frac{s}{2^{2 m} t}\right)^{\frac{n-1}{2}+\frac{3}{4}} \frac{d s}{s} \\
& \leq C \sum_{m=0}^{\infty} 2^{-\frac{m}{2}} \int_{2 t}^{\infty}\left(\frac{t}{s}\right)^{\frac{1}{4}} \frac{d s}{s} \leq C^{\prime}<\infty .
\end{aligned}
$$

Combining the estimates of $\mathrm{I}_{1}, \mathrm{II}_{1,1}, \mathrm{II}_{1,2}$ we obtain (2.5). Hence, the proof of (2.5) is complete. Now, we will start the proof of Theorem 1.1.

Proof of Theorem 1.1. Let us consider the term V. We have that

$$
\left(V^{1 / 2} A^{-1 / 2}\left(I-e^{-t A}\right)\right)^{*}=\left(I-e^{-t A}\right) A^{-1 / 2} V^{1 / 2} .
$$

So the kernel $K_{t}^{*}(y, z)$ of the operator $\left(V^{1 / 2} A^{-1 / 2}\left(I-e^{-t A}\right)\right)^{*}=\left(I-e^{-t A}\right) A^{-1 / 2} V^{1 / 2}$ satisfies the following estimate

$$
\sum_{m=0}^{\infty} 2^{m}\left(2^{m} \sqrt{t}\right)^{n / 2}\left(\int_{2^{m} \sqrt{t} \leq|y-z|<2^{m+1} \sqrt{t}}\left|K_{t}^{*}(y, z)\right|^{2} d z\right)^{1 / 2} \leq C<\infty,
$$

where $C$ is a constant independent of $t$ and $y$.

Finally, from the fact that $|y-z| \geq r_{B}$ for any $y \in B, z \notin 2 B$, the conclusion of (2.3), Lemma 
2.2 and the Hölder inequality, the term $\mathrm{V}$ is dominated as follows,

$$
\begin{aligned}
\mathrm{V} & =\frac{1}{|B|} \int_{B}\left|\left(I-e^{-t_{B} A}\right) T\left(\left(b-b_{B}\right) f_{2}\right)(y)\right| d y \\
& \leq \frac{1}{|B|} \int_{B} \int_{\mathbb{R}^{n} \backslash(2 B)}\left|K_{t_{B}}^{*}(y, z) \|\left(b(z)-b_{B}\right) f(z)\right| d z d y \\
& \leq \frac{1}{|B|} \int_{B} \sum_{m=0}^{\infty}\left(\int_{2^{m} r_{B} \leq|y-z|<2^{m+1} r_{B}}\left|K_{t_{B}}^{*}(y, z)\right|\left|\left(b(z)-b_{B}\right)\right||f(z)| d z\right) d y \\
& \leq C \frac{1}{|B|} \int_{B} \sum_{m=0}^{\infty}\left|2^{m+1} B\right|^{\alpha / n}\|b\|_{\operatorname{Lip}_{\alpha}\left(\mathbb{R}^{n}\right)}\left(\int_{2^{m} r_{B} \leq|y-z|<2^{m+1_{r_{B}}}}\left|K_{t_{B}}^{*}(y, z)\right|^{2} d z\right)^{1 / 2} \\
& \times\left(\int_{2^{m+1}}|f(z)|^{2}\right)^{1 / 2} d y \\
& \leq C \sup _{m \geqslant 0} 2^{-m}\|b\|_{\operatorname{Lip}_{\alpha}\left(\mathbb{R}^{n}\right)} M_{2, \alpha}(f)(x) \\
& \leq C\|b\|_{\operatorname{Lip}_{\alpha}\left(\mathbb{R}^{n}\right)} M_{2, \alpha}(f)(x) .
\end{aligned}
$$

Combining the estimates from I to V, we see that (2.1) holds, which completes the proof of Theorem 1.1.

\section{Acknowledgements}

This work is supported by the National Natural Science Foundation of China (No. 11671031 and No. 11471018), the Fundamental Research Funds for the Central Universities (No. FRF-BR-17-004B), Program for New Century Excellent Talents in University, Beijing Municipal Science and Technology Project (No. Z17111000220000).

\section{References}

[1] B. Bongioanni, E. Harboure and O. Salinas, Commutators of Riesz transforms related to Schrödinger operators, J. Fourier Anal. Appl., 17 (2011), 115-134.

[2] S. Chanillo, A note on commutators, Indiana Univ. Math. J., 31 (1982), 7-16.

[3] R.R. Coifman, R. Rochberg and G. Weiss, Factorzation theorems for Hardy spaces in seval variables, Ann. Math., 103 (1976), 611-635.

[4] S. Janson, Mean oscillation and commutators of singular integrals operators, Ark. Mat., 16 (1978), 263-270.

[5] X.T. Duong and L. Yan, Commutators of Riesz transforms of magnetic Schrödinger operators, Manuscripta Math., 127 (2008), 219-234.

[6] Z. H. Guo, P. T. Li and L. Z. Peng, $L^{p}$ boundedness of commutators of Riesz transform associated to Schrödinger operator, J. Math. Anal. Appl., 341 (2008), 421-432.

[7] P. T. Li and L. Z. Peng, Endpoint estimates for commutators of Riesz transform associated with Schrödinger operators, Bull. Australian Math. Soc., 82 (2010), 367-389.

[8] Y. Liu, Commutators of BMO functions and degenerate Schrödinger operators with certain nonnegative potentials, Monatsh. Math., 165 (2012), 41-56. 
[9] Y. Liu, J. Z. Huang and J. F. Dong, Commutators of Calderón-Zygmund operators related to admissible functions on spaces of homogeneous type and applications to Schrödinger operators, Sci. China. Math., 56 (2013), 1895-1913.

[10] Y. Liu, L. J. Wang and J. F. Dong, Commutators of higher order Riesz transform associated with Schrödinger operators, J. Function Spaces Appl., (2013), Article ID 842375, 15 pages.

[11] Y. Liu and J. L. Sheng, Some estimates for commutators of Riesz transforms associated with Schrödinger operators, J. Math. Anal. Appl., 419 (2014), 298-328.

[12] S. Z. Lu, Q. Wu and D. C. Yang, Boundedness of commutators on Hardy type spaces, Science in China (Series A), 45 (2002), 984-997.

[13] J. M. Martell, Sharp maximal functions associated with approximations of the identity in spaces of homogeneous type and applications, Studia Math., 161 (2004), 113-145.

[14] X. T. Duong, E. M. Ouhabaz and L. X. Yan, Endpoint estimates for Riesz transforms of magnetic Schrödinger operators, Ark. Mat., 44 (2006), 261-275.

[15] Z. W. Shen, Estimates in $L^{p}$ for magnetic Schrödinger operators, Indiana Univ. Math. J., 3 (1996), 817-841.

[16] D. Y. Yang, Commutators of Riesz transforms with Lipschitz functions related to Schrödinger operators, Math. Inequal. Appl., 19 (2016), 173-184. 